\title{
Sugammadex: an opportunity for more thinking or more cookbook medicine?
}

\author{
François Donati PhD MD FRCPC
}

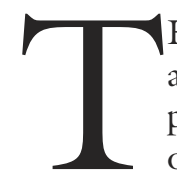

HERE have been virtually no new drugs in anesthesia in the past ten to 15 years, so the prospect of the addition of sugammadex to our carts is being met with great anticipation. Moreover, in contrast to all agents that have been added to our armamentarium in the past few decades, this new drug has a new mechanism of action, quite different from currently available compounds. Briefly, sugammadex is a gamma-cyclodextrin, made up of a ring of eight sugars, to which negatively charged side chains were added for the purpose of binding rocuronium and other steroid-based neuromuscular blocking agents. ${ }^{1,2}$ The first description of sugammadex appeared in the peer-reviewed literature in $2002 .{ }^{2}$ Human trials are underway and the drug should reach market within one to three years.

Sugammadex, previously called Org 25969, has already generated quite a bit of excitement, being qualified as "revolutionary approach", 3 "a milestone", ${ }^{1}$ and an "opportunity to change the practice of anesthesiology". "Such enthusiasm is justified by the real possibility that sugammadex, a selective binding agent, will have none of the severe limitations associated with the use of anticholinesterase agents when reversing neuromuscular blockade. First, these drugs have limited effectiveness, because a ceiling effect is reached when acetyl cholinesterase inhibition is near $100 \%$, and the amount of acetylcholine to compete with the neuromuscular blocking agent can be no more than the quantity released at the nerve terminal. This is why it is recommended to attempt reversal only when significant recovery has already taken place, that is the presence of at least two, and preferably four, twitches in response to train-of-four (TOF) stimulation is documented. ${ }^{5}$ The second problem related to anticholinesterase agents is the cardiovascular sideeffect profile, even with the co-administration of an anticholinergic. By binding to the neuromuscular blocking agent and not to any receptor, sugammadex has the potential to solve the drawbacks of neostigmine and other anticholinesterase agents.

There is a surprising scarcity of hard data related to sugammadex in the peer reviewed literature. At the time of writing (May 2007), a PubMed search for "sugammadex OR Org 25969" returned only 31 articles, with seven being classified as randomized controlled trials. Therefore, there is still considerable speculation about the properties of the drug and many claimed features must be accepted with a healthy dose of caution. Assuming that sugammadex is inert chemically and does not bind to any receptor or any other useful endogenous or exogenous molecule, it could solve the problems of residual paralysis and failed intubation. Even if non-depolarizing neuromuscular blocking agents are administered in doses that seem adequate for the anticipated duration of surgery, residual paralysis is a relatively common problem. ${ }^{6}$ Sugammadex could be a solution to that concern because it is expected that there will always be a dose of the binding agent that will be sufficient for the dose of rocuronium in the body. The same logic can be applied to the problem of failed intubation. If rocuronium is given at induction of anesthesia and the airway cannot be secured, prompt restoration of normal neuromuscular function could be achieved with the appropriate dose of sugammadex. ${ }^{1}$ Succinylcholine could become obsolete, being replaced for the rapid sequence induction scenario by rocuronium, followed by sugammadex as rescue if needed.

The introduction of sugammadex has the potential to change radically our approach to reversal, but will it spare us from the need to understand neuromuscular blockade? Is the era of cookbook blockade management at our doorstep or will our patients be better off if we try to understand what we are doing? An analogy with the introduction of pulse oximetry in anesthesia two decades ago might be interesting.

CAN J ANESTH 2007 / 54: 9 / pp 689-695

From the Department of Anesthesiology, Hôpital Maisonneuve-Rosemont and Université de Montréal, Montréal, Québec, Canada. Address correspondence to: Dr. François Donati, Department of Anesthesiology, Hôpital Maisonneuve-Rosemont, 5415, boul. l'Assomption, Montréal, Québec H1T 2M4, Canada. Phone: 514-252-3426; Fax: 514-252-3542; E-mail: francois.donati@umontreal.ca Disclosure statement: The author has received honoraria and research grants from Organon Inc, the manufacturer of sugammadex. Divulgation: L'auteur a reçu des honoraires et des subventions de recherche d'Organon Inc, le fabricant du sugammadex. 
When non-invasive devices capable of measuring oxygen saturation became available, anesthesiologists did not take long to understand the usefulness of the new equipment. We all knew how oxygen is delivered to the tissues, and the concepts of partial pressure and saturation were familiar to us. This helped with the smooth acceptance of routine pulse oximetry. Today, it is preferable to practice with a mental picture of the oxygen-hemoglobin dissociation curve rather than rely blindly on flow charts or easy recipes designed to fix that number on the monitor. Similarly, future management of neuromuscular blockade will require a mental picture of how rocuronium is distributed in the body, how it works, how sugammadex binds to rocuronium, and where one catches the other. This approach will help choose the right dose for each circumstance. Even if large doses $\left(8-16 \mathrm{mg} \cdot \mathrm{kg}^{-1}\right)$ of sugammadex are essentially free of side-effects, they might be prohibitively expensive for administration on a routine basis, while lower doses $\left(2-4 \mathrm{mg} \cdot \mathrm{kg}^{-1}\right.$, or even $1 \mathrm{mg} \cdot \mathrm{kg}^{-1}$ ) might be adequate in most cases.

The molecular weight of sugammadex is 2,178 Daltons while that of rocuronium bromide is 610 Daltons, and both molecules bind in a 1:1 molar ratio. ${ }^{1,2}$ Thus, slightly less than $4 \mathrm{mg}(3.57 \mathrm{mg}$ to be exact) of sugammadex is required to bind $1 \mathrm{mg}$ of rocuronium. Thus, it is tempting to conclude that effective reversal can be guaranteed by administration of sugammadex $2.4 \mathrm{mg} \cdot \mathrm{kg}^{-1}$ at any time after rocuronium $0.6 \mathrm{mg} \cdot \mathrm{kg}^{-1}$. The situation is not that simple. Binding is not irreversible, and the interaction between the two molecules should be regarded as dynamic, with some complexes being formed while others are being broken down into constituent molecules. The dissociation constant $\left(\mathrm{K}_{\mathrm{d}}\right)$ is reported to be approximately $0.1 \mu \mathrm{M}$ for the rocuronium-sugammadex complex. ${ }^{1,2}$ If $2 \mu \mathrm{M}$ rocuronium, which corresponds to $90-100 \%$ first twitch blockade, ${ }^{7}$ is mixed with $2 \mu \mathrm{M}$ sugammadex, as much as $20 \%(0.4 \mu \mathrm{M})$ of both drugs remain free, while the remaining $80 \%$ is bound. At $0.4 \mu \mathrm{M}$, there is normally no detectable blockade, thanks to the important margin of safety of the neuromuscular junction, except perhaps in the most sensitive individuals, for example those with myasthenia gravis. However, a little extra sugammadex helps a great deal. If instead of mixing $2 \mu \mathrm{M}$ rocuronium with the same concentration of sugammadex, one chooses to give $4 \mu \mathrm{M}$ sugammadex, this would decrease the concentration of free rocuronium to less than $0.1 \mu \mathrm{M}$, a fourfold decrease.

These simple considerations apply to both drugs mixed in a bucket, but the human body is not just a simple bucket. Sugammadex must reach rocuronium where it is, and remove it from the neuromuscular junction. There is no direct evidence of how sugammadex actually achieves this, but an explanation has been offered. When injected, sugammadex binds to rocuronium molecules present in plasma. The resulting decline in free rocuronium in plasma produces a concentration gradient of free rocuronium between interstitial tissue and plasma, favouring transfer of rocuronium into plasma. These fresh rocuronium molecules in plasma are soon bound to sugammadex, and the interstitial tissue, where the neuromuscular junction lies, is rapidly depleted of its rocuronium molecules. Evidence for this process can be found in a study by Epemolu et al., ${ }^{8}$ who demonstrated that, consistent with the above hypothesis, sugammadex was associated with an increase of total (free plus bound) plasma concentration of rocuronium.

The next question has to do with what happens to sugammadex next, and especially to the sugammadexrocuronium complex. Both are excreted unchanged in the urine, ${ }^{8 ; 9}$ and there appears to be no metabolism. Clearance is expected to parallel creatinine clearance, approximately $\mathrm{l}-2 \mathrm{~mL} \cdot \mathrm{kg}^{-1} \cdot \mathrm{min}^{-1}$ in normal subjects. Disposition of sugammadex in patients with diminished or absent renal function is not known. The terminal half-life of sugammadex is one to two hours in subjects with normal renal function. ${ }^{9}$ It is expected that return to a normal response to rocuronium in patients receiving sugammadex will require several half-lives and be sugammadex dose-dependent, but the exact time interval is unknown. This last point is also an argument in favour of using the smallest possible dose of sugammadex, because too much sugammadex on board would limit the options should re-intubation of the trachea or repeat surgery be needed shortly after the end of the case.

So far, studies have focused on the time required for the TOF responses to achieve a ratio $>0.9$ as measured by acceleromyography, after rocuronium followed by sugammadex at various degrees of spontaneous recovery. ${ }^{8-11}$ One may criticize the studies because of the choice of accelerometry, which overestimates the degree of recovery, but this drawback is minor considering how effective sugammadex is compared with either placebo or neostigmine. In most clinical circumstances, the time interval from blockade until full recovery is not expected to be particularly critical. Large doses of sugammadex produce recovery within one to two minutes even after deep blockade, ${ }^{11}$ but there may be little added value to a reduction of recovery time from, say, three to five minutes to one to two minutes. Sugammadex is intended for use in patients with their trachea intubated and their airway secured, 
so a little extra wait until extubation does not jeopardize patient safety. The exception to that time rule is when sugammadex is given as a rescue medication, in the case of failed intubation. In this special case, a twominute recovery time would appear terribly long, and three to five minutes would be unacceptable!

The introduction of sugammadex is expected to induce major changes in the management of neuromuscular blockade. Currently, it is recommended to wait for significant spontaneous recovery until neostigmine can be given safely. This level of spontaneous recovery can be quantified easily by TOF monitoring. When four TOF responses are observed, even if fade is seen, neostigmine is considered reasonably effective, and one expects adequate neuromuscular recovery, now regarded as being a TOF ratio $>0.9$, to occur within five to $15 \mathrm{~min}$, with no recurarization. ${ }^{5}$

The rules will change markedly with the introduction of sugammadex. The requirement to obtain some degree of spontaneous recovery will no longer be present. However, the sugammadex dose required depends heavily on the intensity of the block: when two twitches have reappeared, $1-2 \mathrm{mg} \cdot \mathrm{kg}^{-1}$; at deeper blockade, when the post-tetanic count is only 2, 2-4 $\mathrm{mg} \cdot \mathrm{kg}^{-1}$; and immediately after rocuronium, for rescue purposes, 8-16 mg. $\mathrm{kg}^{-1}$. 1,10,11 These initial recommendations may change as more data become available. This dose-dependency can be readily explained by the need to bind more rocuronium in plasma as blockade becomes deeper. Thus, even after the introduction of sugammadex, neuromuscular monitoring will be useful, allowing the right dose to be chosen. The alternative would be to give a large sugammadex dose for all cases, a more expensive course of action than monitoring.

The other question that needs to be answered relates to the possibility of re-paralysis. Such an event has already been reported, ${ }^{12}$ and the phenomenon can be understood as follows. If the dose of sugammadex given is just enough to capture most of the rocuronium in plasma, then there will be sufficient movement of rocuronium away from the neuromuscular junction down the concentration gradient of free drug into plasma. This may produce full return of neuromuscular function. However, with time, more rocuronium molecules will be transferred from peripheral tissue into plasma, and there will no longer be enough free sugammadex molecules available. The free rocuronium will then have access to the neuromuscular junction, where blockade can ensue. It is uncertain at this time what the minimum sugammadex dose not associated with this recurarization is, or how long patients need to be monitored to avoid this effect. Another solution, which has not been tested, is to administer sugammadex in divided doses: a first injection to achieve immediate recovery, and a second to make sure there is no recurarization.

When sugammadex becomes available, concerns about reversal of blockade at the end of a case will be diminished. Therefore, anesthesiologists may be tempted to give larger doses of rocuronium than they do now, thus increasing the cost of inducing paralysis as well as the cost of reversing it. There could be benefits to this new approach: better intubating conditions, less delay between induction and laryngoscopy, less desaturation, less airway trauma, better surgical conditions, fewer respiratory problems at emergence, less residual paralysis. However, there are some dangers. There could be a greater incidence of awareness, because total absence of movement may mask insufficient anesthesia and analgesia. In this context it should be realized that administration of sugammadex is frequently accompanied by manifestations of inadequate anesthesia, even if the background anesthetic is unchanged. ${ }^{11}$ Also, the problem of managing the airway after sugammadex has been given, for instance if a repeat procedure needs to be performed, is not settled. Perhaps there will be a (small) role for succinylcholine after all. But the biggest danger is to forget to monitor and understand neuromuscular blockade. The tendency to adopt a "one dose fits all" approach for both rocuronium and sugammadex is likely to become expensive and contrary to the patient's best interests.

\section{Le sugammadex: une occasion de réfléchir ou de suivre la recette?}

Il n'y a pratiquement pas eu de nouvelles molécules en anesthésie depuis 10 ou 15 ans, de sorte que la perspective de voir s'ajouter le sugammadex à la liste de nos médicaments est accueillie avec trépidation. De plus, contrairement aux autres agents qui sont apparus au cours des dernières décennies, le nouveau venu a un nouveau mécanisme d'action, différent de ce que l'on connait. En bref, le sugammadex est une gammacyclodextrine, constituée d'un anneau de huit sucres, auquel on a ajouté des chaînes latérales chargées négativement pour se lier au rocuronium et aux autres curares à noyau stéroïdien. ${ }^{1,2}$ Le sugammadex a été 
décrit pour la première fois dans la littérature scientifique en 2002. ${ }^{2}$ Des essais cliniques sur les humains sont en cours et la molécule devrait être disponible en clinique d'ici un à trois ans.

Le sugammadex, appelé auparavant ORG 25969, a été l'objet de bien des espoirs et a été qualifié d'approche révolutionnaire, ${ }^{3}$ d'événement marquant $^{1}$ et d'occasion de changer la pratique de l'anesthésiologie. ${ }^{4}$ Cet enthousiasme est justifié dans la mesure où le sugammadex, en se liant de façon spécifique, pourrait ne posséder aucun des inconvénients majeurs que possèdent les anticholinestérasiques utilisés pour neutraliser le bloc neuromusculaire. Tout d'abord, ces derniers ont une efficacité limitée, parce qu'un effet plafond s'observe à des doses correspondant à une inhibition de l'acétyl cholinestérase voisine de $100 \%$. En effet, la quantité d'acétylcholine disponible pour interagir de façon compétitive avec le curare ne peut dépasser celle qui est libérée par la terminaison nerveuse. C'est pourquoi on recommande de tenter une décurarisation pharmacologique seulement lorsqu'il y a récupération significative, soit la présence manifeste d'au moins deux, voire quatre réponses à la stimulation en train-de-quatre. ${ }^{5}$ Un autre problème que cause l'administration d'anticholinestérasique est la survenue d'effets cardiovasculaires, même avec l'ajout d'un anticholinergique. Puisque le sugammadex se lie au curare sans interagir avec un récepteur, le sugammadex pourrait être une solution de rechange à la néostigmine et aux autres anticholinestérasiques.

Pourtant, il existe peu de données probantes au sujet du sugammadex dans la littérature scientifique. Au moment d'écrire ces lignes (mai 2007), une recherche PubMed utilisant les mots-clés «sugammadex OU Org 25969 » a permis de trouver que 31 articles, dont sept se qualifiaient comme études randomisées contrôlées. Ainsi, il existe de nombreuses zones d'ombre quant aux propriétés de cette molécule et plusieurs des caractéristiques qu'on lui attribue doivent être accueillies avec une bonne dose de scepticisme. En supposant que le sugammadex est chimiquement inerte et qu'il n'interagit avec aucun récepteur ou aucune molécule endogène ou exogène utile, il pourrait résoudre les problèmes de curarisation résiduelle et d'échec à l'intubation. Même en dosant les curares non dépolarisants de sorte que leur durée d'action ne dépasse pas celle qui est prévue pour la chirurgie, la curarisation résiduelle reste un problème assez fréquent. ${ }^{6}$ Le sugammadex pourrait constituer une solution parce qu'il existerait toujours une dose suffisante pour neutraliser toutes les molécules de rocuronium présentes dans l'organisme. Le même raisonnement s'appliquerait au cas d'échec à l'intubation. Si l'on donne du rocuronium à l'induction de l'anesthésie et que l'intubation est impossible, le patient pourrait retrouver rapidement une fonction neuromusculaire normale suite à une dose adéquate de sugammadex. ${ }^{1}$ La succinylcholine deviendrait alors désuète et serait remplacée pour une induction avec séquence rapide par du rocuronium, suivi si nécessaire par du sugammadex donné comme mesure de sauvetage.

L'arrivée du sugammadex pourrait changer profondément notre attitude vis-à-vis la neutralisation $\mathrm{du}$ bloc neuromusculaire, mais serions-nous bien avisés d'en oublier la physiologie ? Entrons-nous dans une logique où il suffit d'adopter quelques recettes ? Au contraire, nos patients se porteront-ils mieux si nous essayons de comprendre ce que nous faisons ? L'analogie avec l'arrivée de la saturométrie il y a une vingtaine d'années pourrait être riche d'enseignements. Lorsque des appareils capables de mesurer la saturation en oxygène de façon non invasive sont devenus disponibles, les anesthésiologistes n'ont pas perdu de temps à comprendre l'utilité de ce nouveau matériel. Nous savions tous alors comment l'oxygène se rend aux tissus et nous maitrisions les concepts de pression partielle et de saturation. Ces connaissances ont facilité l'acceptation en douceur de la saturométrie comme monitorage standard. En pratique courante, il est préférable de se faire une représentation mentale de la courbe de dissociation de l'hémoglobine plutôt que de se fier de façon aveugle à des organigrammes ou des recettes faciles destinées à normaliser le chiffre affiché sur le moniteur. De même, la prise en charge de la curarisation dans l'avenir reposera sur une représentation mentale de la distribution du rocuronium dans l'organisme, de son mécanisme d'action, des interactions sugammadex-rocuronium et du lieu de rencontre de ces deux molécules. Cette approche permettra de choisir la bonne dose pour chaque situation. Même si les doses élevées de sugammadex (8-16 $\mathrm{mg} \cdot \mathrm{kg}^{-1}$ ) semblent dépourvues d'effets secondaires significatifs, leur administration systématique pourrait être coûteuse alors que des doses plus modestes (2-4 $\mathrm{mg} \cdot \mathrm{kg}^{-1}$, voire $1 \mathrm{mg} \cdot \mathrm{kg}^{-1}$ ) pourraient faire l'affaire dans la plupart des cas.

Le poids moléculaire du sugammadex est de 2178 Daltons tandis que celui du rocuronium est de 610 Daltons et les deux molécules se lient dans un rapport 1:1. ${ }^{1,2}$ Ainsi, on a besoin d'un peu moins de $4 \mathrm{mg}$ de sugammadex (plus précisément de $3,57 \mathrm{mg}$ ) pour lier $\mathrm{l} \mathrm{mg}$ de rocuronium. On pourrait alors être porté à conclure qu'une neutralisation efficace de la curarisation serait garantie par l'administration de sugammadex, 2,4 $\mathrm{mg} \cdot \mathrm{kg}^{-1}$, n'importe quand après l'injection de rocuronium, $0,6 \mathrm{mg} \cdot \mathrm{kg}^{-1}$. Les choses ne sont pas 
si simples. Le lien n'est pas irréversible et l'interaction entre les deux molécules doit être considérée comme dynamique, avec formation de nouveaux complexes alors que d'autres liens se rompent et que les deux molécules retrouvent leur identité. La constante de dissociation $\left(\mathrm{K}_{\mathrm{d}}\right)$ est évaluée à $0,1 \mu \mathrm{M}$ pour la réaction rocuronium-sugammadex. ${ }^{1,2} \mathrm{Si}$ du rocuronium, à la concentration de $2 \mu \mathrm{M}$, ce qui correspond à un bloc de $90-100 \%$ de la première réponse au train-de-quatre, ${ }^{7}$ est mélangé à $2 \mu \mathrm{M}$ de sugammadex, la concentration libre de chacun des médicaments atteint $20 \%$ $(0,4 \mu \mathrm{M})$, tandis que le reste $(80 \%)$ est lié. À $0,4 \mu \mathrm{M}$, on ne détecte aucune curarisation, à cause de la marge de sécurité importante à la jonction neuromusculaire, sauf chez les sujets particulièrement sensibles comme ceux atteints de myasthénie grave. Toutefois, un peu plus de sugammadex améliore grandement les choses. $\mathrm{Si}$, au lieu de mélanger du rocuronium, $2 \mu \mathrm{M}$, avec la même concentration de sugammadex, on décidait d'y mettre $4 \mu \mathrm{M}$ de sugammadex, la concentration de rocuronium libre serait réduite de 4 fois, à $0,1 \mu \mathrm{M}$.

Ces calculs simples s'appliquent à la situation où les deux molécules sont mélangées dans une éprouvette, mais le corps humain n'est pas une simple éprouvette. Le sugammadex doit atteindre le rocuronium où il se trouve et l'arracher à la jonction neuromusculaire. Sans avoir de preuve directe du mécanisme exact d'action, une explication du processus a été proposée. Après injection, le sugammadex se lie aux molécules de rocuronium qui se trouvent dans le plasma, réduisant ainsi le nombre de molécules libres. Un gradient de concentration est créé pour le rocuronium libre entre le compartiment interstitiel et le plasma, favorisant un transfert de rocuronium vers le plasma. Le sugammadex se lie immédiatement à ces molécules de rocuronium qui arrivent et le compartiment interstitiel, là où se trouve la jonction neuromusculaire, se vide rapidement de son rocuronium. Des données appuyant cette hypothèse ont été fournies par Epemolu et coll., ${ }^{8}$ qui ont établi que l'injection de sugammadex était suivie d'une augmentation de la concentration plasmatique totale (libre plus liée) de rocuronium.

Reste à savoir ce qui arrive ensuite au sugammadex et surtout au complexe sugammadex-rocuronium. Les deux sont éliminés inchangés dans l'urine ${ }^{8,9}$ et il ne semble y avoir aucun métabolisme. La clairance devrait donc suivre la clairance de la créatinine, soit environ $1-2 \mathrm{~mL} \cdot \mathrm{kg}^{-1} \cdot \mathrm{min}^{-1}$ chez les sujets sains. Le mode d'élimination du sugammadex chez les patients avec une insuffisance rénale est inconnu. La demi-vie d'élimination du sugammadex est d'une à deux heures chez les sujets dont la fonction rénale est normale. ${ }^{9}$ On s'attend à ce qu'il faille plusieurs demi-vies pour que la réponse au rocuronium revienne à la normale chez les patients qui ont reçu du sugammadex et que cet intervalle dépende de la dose de sugammadex, mais on ne connait pas le chiffre exact. Cet aspect de la question est aussi un argument en faveur de l'utilisation de la plus petite dose efficace de sugammadex, parce qu'un excès de sugammadex pourrait limiter les possibilités si une intubation trachéale ou une deuxième intervention chirurgicale devenaient nécessaires peu après une chirurgie.

Jusqu'à présent, les études ont porté principalement sur le temps qu'il fallait pour atteindre un rapport quatrième/première réponse $>0,9$ mesuré par accéléromyographie, après une injection de sugammadex donnée à différents niveaux de récupération spontanée suite à du rocuronium..$^{8-11}$ Dans ces études, on peut critiquer le choix de l'accélérométrie, qui surestime le niveau de récupération, mais ce problème est mineur quand on constate l'efficacité remarquable du sugammadex comparée soit au placebo, soit à la néostigmine. En pratique, l'intervalle entre la curarisation et la récupération complète ne devrait pas être d'une importance cruciale. Des doses élevées de sugammadex produisent une récupération complète en une ou deux minutes même si la curarisation est profonde, ${ }^{11}$ mais il semble futile de rechercher à réduire cet intervalle de, par exemple, trois ou cinq minutes jusqu'à une ou deux minutes. En effet, on prévoit utiliser le sugammadex surtout chez les patients dont la trachée est intubée chez qui on contrôle les voies aériennes, de sorte qu'une attente de courte durée ne met pas en danger la sécurité du patient. L'exception à cette règle est lorsque le sugammadex est donné en situation de sauvetage, suite à un échec à l'intubation. Dans ce cas particulier, une récupération en deux minutes semblerait terriblement longue et une attente de trois ou cinq minutes serait inacceptable.

L'arrivée du sugammadex pourrait déclencher des changements majeurs dans la prise en charge de la curarisation. Actuellement, on recommande d'attendre un niveau suffisant de récupération spontanée, mesurée facilement par monitorage du trainde-quatre, avant de donner de la néostigmine de façon sécuritaire. Lorsque l'on observe quatre réponses, même si elles sont inégales, la néostigmine peut être considérée assez efficace. On s'attend alors à une récupération satisfaisante de la fonction neuromusculaire, maintenant considérée comme étant un rapport quatrième/première réponse $>0.9$, en cinq à $15 \mathrm{~min}$, sans recurarisation. ${ }^{5}$

La donne changera profondément avec l'arrivée du sugammadex, parce qu'il ne sera plus nécessaire d'attendre la venue d'une récupération spontanée. 
Toutefois, la dose de sugammadex dépend fortement de l'intensité de la curarisation : $1-2 \mathrm{mg} \cdot \mathrm{kg}^{-1}$ lorsque deux réponses au train-de-quatre sont visibles ; $2-4$ $\mathrm{mg} \cdot \mathrm{kg}^{-1}$ si la curarisation est plus profonde, avec un décompte post-tétanique de 2 ; et enfin $8-16 \mathrm{mg} \cdot \mathrm{kg}^{-1}$ immédiatement après injection de rocuronium, dans des situations de sauvetage..$^{1,10,11}$ Ces premières recommandations pourraient changer à la lumière de données à venir. On peut expliquer la relation entre la dose de sugammadex et l'intensité de la curarisation par la plus grande quantité de rocuronium à lier dans le plasma quand le bloc neuromusculaire est profond. Ainsi, même lorsque le sugammadex sera disponible, le monitorage de la jonction neuromusculaire sera utile, parce qu'il facilitera le choix de la bonne dose. L'alternative serait d'administrer une dose élevée de sugammadex dans tous les cas, une démarche plus coûteuse que le monitorage.

Par ailleurs, on doit se poser la question de la possibilité de recurarisation. Ce phénomène a déjà été documenté ${ }^{12}$ et il pourrait s'expliquer comme suit. Supposons que la dose de sugammadex est tout juste assez pour se lier à presque tout le rocuronium présent dans le plasma. Il y aura alors transfert de rocuronium de la jonction neuromusculaire vers le plasma, en suivant un gradient de concentration favorable, ce qui pourrait permettre une récupération de la fonction neuromusculaire. Toutefois, il suffirait d'un peu plus de temps pour que d'autres molécules de rocuronium se mobilisent des tissus périphériques vers le plasma, où il n'y a plus assez de sugammadex non lié. Le rocuronium libre aurait alors accès à la jonction neuromusculaire, pouvant produire une curarisation. On ne sait pas encore quelle est la dose minimum de sugammadex qui prévient cette recurarisation ni quelle est la durée de monitorage nécessaire pour éviter cet effet. Une autre solution, qui n'a pas encore été mise à l'épreuve, consisterait en l'administration de deux doses successives de sugammadex : la première pour obtenir une récupération immédiate, la seconde pour éviter une recurarisation possible.

Avec le sugammadex, les difficultés à décurariser à la fin de l'intervention seront moindres, de sorte que les anesthésiologistes pourraient ne pas résister à la tentation de donner des doses plus élevées de rocuronium que maintenant, augmentant ainsi le coût de la curarisation et le coût de la décurarisation. Cette façon de faire pourrait avoir des avantages : de meilleures conditions d'intubation, un intervalle réduit entre l'induction et la laryngoscopie, moins de désaturation, moins de traumatisme des voies aériennes, moins de problèmes respiratoires à l'émergence, moins de curarisation résiduelle. Toutefois, il y a quelques dangers. Il pourrait y avoir plus d'éveil intraopératoire, puisque l'absence totale de mouvement pourrait masquer une anesthésie et une analgésie insuffisantes. À ce propos, soulignons que l'administration de sugammadex est souvent accompagnée de manifestations d'une anesthésie insuffisante, même si l'anesthésique de base reste inchangé. ${ }^{11}$ De plus, le problème de la réintubation après administration de sugammadex, par exemple si une deuxième intervention s'avère nécessaire, n'a pas été résolu. Peut-être restera-t-il une (petite) place à la succinylcholine après tout ? Mais le danger le plus grave est d'oublier le monitorage et de ne plus comprendre la curarisation. La tentation de se confiner à une conduite comportant une seule dose de rocuronium et une seule dose de sugammadex pour toutes les situations pourrait devenir coûteuse et contraire aux meilleurs intérêts de nos patients.

\section{References}

1 Naguib M. Sugammadex: another milestone in clinical neuromuscular pharmacology. Anesth Analg 2007; 104: 575-81.

2 Bom A, Bradley M, Cameron K, et al. A novel concept of reversing neuromuscular block: chemical encapsulation of rocuronium bromide by a cyclodextrin-based synthetic host. Angew Chem Int Ed Engl 2002; 41: 266-70.

3 Kopman AF. Sugammadex: a revolutionary approach to neuromuscular antagonism. Anesthesiology 2006; 104: 631-3.

4 Miller RD. Sugammadex: an opportunity to change the practice of anesthesiology? Anesth Analg 2007; 104: 477-8.

5 Kim KS, Cheong MA, Lee HJ, Lee JM. Tactile assessment for the reversibility of rocuronium-induced neuromuscular blockade during propofol or sevoflurane anesthesia. Anesth Analg 2004; 99: 1080-5.

6 Naguib M, Kopman AF, Ensor JE. Neuromuscular monitoring and postoperative residual curarisation: a meta-analysis. Br J Anaesth 2007; 98: 302-16.

7 Plaud B, Proost JH, Wierda JM, Barre J, Debaene B, Meistelman $C$. Pharmacokinetics and pharmacodynamics of rocuronium at the vocal cords and the adductor pollicis in humans. Clin Pharmacol Ther 1995; 58 : 185-91.

8 Epemolu O, Bom A, Hope F, Mason R. Reversal of neuromuscular blockade and simultaneous increase in plasma rocuronium concentration after the intravenous infusion of the novel reversal agent Org 25969. Anesthesiology 2003; 99: 632-7.

9 Gijsenbergh F, Ramael S, Houwing N, van Iersel $T$. First human exposure of Org 25969, a novel agent to reverse the action of rocuronium bromide. 
Anesthesiology 2005; 103: 695-703.

10 Sorgenfrei IF, Norrild K, Larsen PB, et al. Reversal of rocuronium-induced neuromuscular block by the selective relaxant binding agent sugammadex: a dosefinding and safety study. Anesthesiology 2006; 104 : 667-74.

11 Sparr HJ, Vermeyen KM, Beaufort AM, et al. Early reversal of profound rocuronium-induced neuromuscular blockade by sugammadex in a randomized multicenter study: efficacy, safety, and pharmacokinetics. Anesthesiology 2007; 106: 935-43.

12 Eleveld DJ, Kuizenga K, Proost JH, Wierda JM. A temporary decrease in twitch response during reversal of rocuronium-induced muscle relaxation with a small dose of sugammadex. Anesth Analg 2007; 104: 582-4. 